\title{
Milk, coronary disease and mortality
}

Milk has long been under suspicion as an important factor in coronary heart disease because of its relatively high content of saturated fat and numerous people and expert groups have spoken and written in condemnation of milk and have recommended that only skimmed or semiskimmed milk is drunk - even by children.

Many mechanisms have however been appealed to in attempts to explain the supposed harm of milk. Thus it has been argued that its high calcium content, together with an enhancement of the uptake of calcium from other foods by the lactose in milk, could increase arterial calcification, leading to myocardial ischaemia. ${ }^{1}$ On the other hand, animal proteins contribute to homocysteine and milk, unlike meat, contains little of the B vitamins needed for the metabolism of homocysteine. ${ }^{2}$ Milk is also low in copper, an essential element in many enzymes, and the lactose in milk interferes with the absorption of copper from other food sources. ${ }^{3}$ Chronic infections from milk borne bacteria, or algae, have been suggested as a contributory cause of atherosclerosis. ${ }^{4}$ Both phytoestrogens ${ }^{5}$ and xanthine oxidase ${ }^{6}$ are present in milk and both may enhance atheromatous plaque formation. The prevalence of lactose malabsorption within different communities matches both milk consumption and ischaemic heart disease mortality, ${ }^{7}$ and antibodies to heated milk proteins have been shown to be raised in patients with ischaemic heart disease. ${ }^{8}$ Finally, Popham et $a l^{9}$ pointed out that there is a negative correlation between milk consumption and the intake of alcohol and subjects with a high milk intake may therefore deny themselves some of the cardiovascular protection from alcohol.

On the other hand, mechanisms that are of possible benefit in relation to vascular disease have been suggested for milk. Protection from calcium has been suggested. ${ }^{10}$ The apparent paradox thought to be raised by the Masai, with their high milk intake but low coronary heart disease rates, has been explained by milk having an unknown hypocholesterol factor. ${ }^{11}$ On page 379 Ness et al make the further suggestion that increased growth in childhood consequent on a high milk intake, is associated with protection later against vascular disease.

It is a relief to turn from these varied and conflicting hypotheses-however ingenious - to evidence from an observational study. Ness et al report results from a large prospective study in which information on milk consumption was obtained from over five and a half thousand men aged 35 to 64 years, and is now related to deaths during the following 25 years.

The best evidence of course comes from randomised trials, and so far the relevance of milk consumption to cardiovascular death has not been tested in an RCT. A very small crossover trial in children showed that cow's milk had a greater cholesterolaemic effect than soy protein milk. ${ }^{12}$ Another RCT in 500 infants that tested the withholding of cow's milk and the provision of soya formula milk feed gave no evidence of any difference in asthma or other allergic condition. ${ }^{13}$ An RCT that is referred to by Ness et al is however of more direct relevance to cardiovascular disease in that it showed that the provision of a small milk supplement each school day enhanced the growth of children in large families of lower social class. ${ }^{14}$

The conduct of an RCT of adequate size and duration to test the effect on coronary disease of the avoidance of cow's milk in adults would be a formidable undertaking. The level of conviction in the general community that fat from milk and other dairy products is a major cause of heart disease should however make the enlisting of subjects into an RCT relatively easy. On the other hand, the number of possible mechanisms in which milk may be involved would make it difficult to decide how complete milk avoidance would have to be, and for how long before an effect on cardiovascular incidence might be detected.

In this situation, evidence from observational studies is probably the best we will ever have. The paper by Ness et $a l$ is therefore of particular interest. They find no evidence of an association between milk consumption and either cholesterol of triglyceride level. Nor do they find that milk consumption is associated with an increased risk of death, either from coronary heart disease or from all causes. Indeed the relative risk of death from any cause, after adjustment for confounding by a number of possible factors, is significantly reduced in the men with the highest intakes (more than one third of a pint (0.57 litres)) of milk per day, and the risk of death from coronary disease is also reduced $(\mathrm{p}=0.05)$.

The authors are cautious and admit that there might still be residual confounding of the relations of interest. Milk drinking was certainly associated with a number of other factors indicative of better health and greater care, yet it would seem most unlikely that any harmful effect could have been missed.

The overall conclusion of these authors: that the "notion" that regular consumption of milk is hazardous to health is not supported by their data, is consistent with studies already reported in the literature. They quote seven large prospective studies in none of which was there any significant excess in coronary disease incidence. Nor in several of the studies, was there any evidence of any increase in stroke risk. Indeed several other studies have suggested an inverse relation between fat intake and the incidence of stroke.

Yet again, the superiority of epidemiology over conjecture about possible mechanisms is demonstrated.

PETER ELWOOD

Department of Epidemiology and Community Health, University of Wales College of Medicine, University of Wales Hospital, Cardiff CF14 4XN, UK(pelwood@doctors.org.uk)

1 Seely S. Possible connection between milk and coronary heart disease: the calcium hypothesis. Med Hypotheses 2000;54:701-3.

2 Grant WB. Milk and other dietary influences on coronary heart disease. Alternative Medicine Review 1998;3:281-94.

3 Strain JJ. Milk consumption, lactose and copper in the aetiology of Strain JJ. Milk consumption, lactose and copper in the
ischaemic heart disease. Med Hypotheses 1988;25:99-101.

4 Rank P. Milk and atherosclerosis. Med Hypotheses 1986;20:317-38.

5 Seely S. The possible connection between phytoestrogens, milk and coronSeely S. The possible connection between phytoes
ary heart disease. Med Hypotheses 1982;8:349-54.

6 Ho CY, Clifford AJ. Bovine milk xanthine oxidase, blood lipids and coronary plaques in rabbits. $\mathcal{F}$ Nutr $1977 ; 107: 758-66$

Segall JJ. Hypothesis is lactose a dietary risk factor for ischaemic heart disease? Int f Epidemiol 1980;9:271-6.

8 Davies DF. Hypothesis. An immunological view of atherogenesis. Fournal of Atherosclerosis Research 1969;10:253-9.

9 Popham RE, Schmidt W, Israel Y. Variation in mortality from ischaemic heart disease in relation to alcohol and milk consumption. Med Hypotheses 1983;12:321-9.

10 Knox EG. Food and diseases. Br f Prev Soc Med 1977;31:71-80.

11 Gibney MJ, Burstyn PG. Milk, serum cholesterol and the Masai. A hypothesis. Atherosclerosis 1980;35:339-43.

12 Jaques $\mathrm{H}$, Laurin D, Moorjani S, et al. Influence of diets containing cow's milk or soy protein neverage onm plasma lipids in children with familial hilk or soy protein neverage onm plasma lipids in children with

13 Merrett TG, Burr ML, Butland BK, et al. Infant feeding and allergy: Merrett TG, Burr ML, Butland BK, et al. Infant feeding and allergy:
12 -month prospective study of 500 babies born into allergic families. Ann 12-month prospective

14 Baker IA, Elwood PC, Hughes J, et al. A randomised controlled trial of the effect of the provision of free school milk on the growth of children. $\mathcal{F}$ Epidemiol Community Health 1980;34:31-4. 